\title{
Electron-ion interaction in doped conducting polymers
}

\author{
V. N. Prigodin,,${ }^{1,2}$ F. C. Hsu, ${ }^{1}$ J. H. Park, ${ }^{1}$ O. Waldmann, ${ }^{1}$ and A. J. Epstein ${ }^{1,3}$ \\ ${ }^{1}$ Department of Physics, The Ohio State University, Columbus, Ohio 43210-1117, USA \\ ${ }^{2}$ Ioffe Institute, St. Petersburg, 194021 Russia \\ ${ }^{3}$ Department of Chemistry, The Ohio State University, Columbus, Ohio 43210-1185, USA
}

(Received 6 September 2007; published 3 July 2008)

\begin{abstract}
The discovery of electric-field effect for conducting polymers in transistor structures aroused a number of questions about structure, mechanism of charge transport, and a role of ions in conducting polymers. We present here the model of an electrochemical transistor whose resistance is governed by the gate potential through bulk ionic charging/discharging of the conducting polymer-based active channel. The predicted $I(V)$ characteristics do not agree with the measured experimental dependencies for highly doped conducting polymer-based transistors. We suggest that the observable electric-field effect in conducting polymers is related to their structural peculiarities. The large free volume within the conductive polymer chain network enables ions to easily move into and out of the polymers. The main effect of ion insertion is breaking of the percolation network for the conductivity by removing critical hoping sites and, as a result, producing a conductornonconductor transition.
\end{abstract}

DOI: 10.1103/PhysRevB.78.035203

PACS number(s): 85.30.Tv, 82.35.Cd, 73.61.Ph, 66.30.Dn

\section{INTRODUCTION}

Heavily doped conjugated polymers are electrical conductors of interest for both fundamental science and application in electronic devices. Due to their chain morphology the electric response of polymers exhibits unusual features that do not have analogies among conventional conductors. ${ }^{1}$ In the absence of doping the conjugated polymers are onedimensional Peierls insulators with electronic charge-density modulation along chains in the form of single and double bonds. ${ }^{2}$ The Coulomb electron-electron interaction also contributes to the Peierls gap of the electronic spectrum. Heeger, MacDiarmid, and Shirikawa have shown that extra electrons chemically or electrochemically can be introduced into or removed from the polymer chains. ${ }^{1}$ At light doping, the extra charges are not free electrons or holes as in conventional semiconductors, but they are the strongly bound with the Peierls distortion of the backbone. ${ }^{2}$ For a conjugated polymer such as trans-polyacetelyne with a degenerate ground state upon interchange of single and double bonds, those bound states are classified as neutral solitons with spin or charged spinless solitons. In the absence of degeneracy, threedimensional Coulomb and interchain interactions convert solitons into polarons and bipolarons.

Heavy doping of conjugated polymers lifts the Peierls alternation and entirely suppresses the gap in the electronic spectrum leading to a profound increase of the roomtemperature conductivity, $\sigma_{\mathrm{RT}} \cdot{ }^{1-4}$ The $\sigma_{\mathrm{RT}}$ of the first doped polymers had modest values and turned nonmetallic with cooling. ${ }^{5,6}$ This dc-transport behavior of poorly conducting polymers is similar to that observed in amorphous semiconductors ${ }^{7}$ and dirty metals, ${ }^{8}$ although some differences due to the chain morphology are observed. A more adequate description of charge transport of poorly conducting polymers may be obtained within the quasi-onedimensional models with weak interchain hopping or by using a fractal chain models with reduced transverse dimensionality. ${ }^{9,10}$
In the last decade, remarkable progress was made in stabilizing the highly conducting state in heavily doped conjugated polymers. A finite residual conductivity at very low temperatures down to $10 \mathrm{mK}$ was successfully attained for heavily doped polymers ${ }^{11}$ (for recent reviews see, e.g., Refs. 12 and 13). The experiments also indicate that in spite of the large value of $\sigma_{\mathrm{RT}}$ and finite residual conductivity, these highly conducting polymers are still far from being typical metals, e.g., the conductivity of most of samples still continues to decrease with decreasing temperature. Therefore, it was suggested ${ }^{11-13}$ that this latest generation of conducting polymers is close to insulator-metal transition (IMT) which, in according with the general consensus, is an Anderson type of the disorder-driven localization-delocalization transition. ${ }^{8}$ Through a small increase of disorder, e.g., with aging, the polymers often can be driven into the insulator state. The dielectric phase (doped polymer that becomes insulating at low temperatures) can be converted back into the metallic state by, for example, applying pressure. ${ }^{13}$ At the same time, the $\sigma_{\mathrm{RT}}$ varies only slightly with pressure.

The principal advance in the understanding of metallic state in conducting polymers was obtained by studying the microwave and optical conductivity. ${ }^{12,14}$ It was found that the low-frequency electromagnetic response of the metallic samples when analyzed in terms of a Drude metal is provided by an extremely small fraction $\left(\sim 10^{-3}\right)$ of total number of available electrons with very high mobility or very long relaxation time $\left(\sim 10^{-12} \mathrm{~s}\right)$. These data initiated the proposal on a new mechanism of charge transport and a new type of IMT for highly conducting doped polymers. ${ }^{15}$ From structural study, it is well established that the conjugated polymers are strongly inhomogeneous materials. ${ }^{16}$ In "cooked spaghettilike" polymer chain media, one can distinguish the "crystalline" regions within which polymer chains are dense and well packed. Outside those crystalline domains, chains are poorly ordered with lower density. When the polymers approach the IMT, the electron delocalization first happens inside these crystalline regions (metallic 


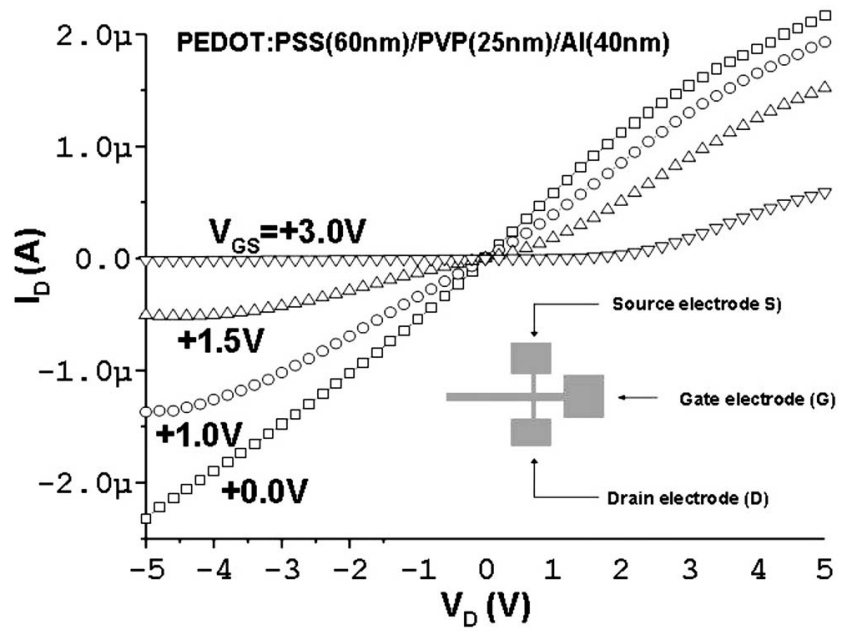

FIG. 1. The drain-source current as the function of drain voltage $\left(V_{G}\right.$ varying between 0 and $3 \mathrm{~V}$ ) of a thin PEDOT:PSS/PVP film.

grains). The wave functions of electronic states in the amorphous region stay strongly localized. ${ }^{15}$

In this chain-linked granular model, the metallic grains with delocalized charge carriers remain spatially separated by amorphous regions, and, therefore, direct electron transitions between grains are suppressed exponentially with increasing grain separation. The intergrain charge transfer is possible because of the resonance tunneling through localized states in amorphous media, whose energy is close to the Fermi level. The resonance hopping among metallic grains can explain the low-frequency anomaly of electromagnetic response in the metallic phase. ${ }^{11,12}$ The low concentration of charge carriers participating in dc transport corresponds to the small probability of finding those resonance states and the long relaxation time of electric response is related to the narrow width of the resonance levels.

The present paper addresses another interesting phenomenon observed in the conducting polymers, an electric-field effect. It was demonstrated ${ }^{17,18}$ that conducting polymers may be used as active elements in a transistor structure. An example is shown in the inset of Fig. 1. The conducting polymer poly(3,4-ethylenedioxythiophene) doped with poly(styrene sulfonic acid) (PEDOT:PSSA) has most often been used for these transistors, but the field effect is also observed in other conducting polymers, including doped polyaniline, doped polypyrrole and their doped copolymers. ${ }^{19}$ All processing and materials handling are made in an ambient atmosphere. Early versions of the device, including a $H$-shaped source/drain bridge and the $T$-shaped gate, were coated on a transparency film, using PEDOT:PSSA for the source-active channel-drain and also for the gate electrode. Polyvinylphenol (PVP) was used as the insulator between the active channel and the gate. The thickness of the PEDOT:PSSA film for these devices typically is $60 \mathrm{~nm}$ and thickness of insulator PVP layer between the gate and polymer film typically is 25 $\mathrm{nm}$.

$I-V$ characteristics of this conducting polymer-based transistor are shown in Fig. 1 at several gate voltages. At floating gate potential, the source-drain current $I_{\mathrm{SD}}$ linearly increases with source-drain voltage $V_{\mathrm{SD}}$ and the corresponding $\sigma_{\mathrm{RT}}$ of
PEDOT/PSSA is $26 \mathrm{~S} / \mathrm{cm}$. As it is shown in Fig. 1, the $I_{\mathrm{SD}}$ current decreases with positive gate voltage $V_{G}$ and the cutoff voltage for $I_{\mathrm{SD}}$ is approximately around $3 \mathrm{~V}$. The ratio $I_{\mathrm{ON}} / I_{\mathrm{OFF}}$ is 100 for the $V_{G}=3 \mathrm{~V}$ and reaches up to $10^{4}$ in other devices at higher gate voltage. Replacement of the doped conducting polymer in the architecture of Fig. 1 with a metal such as copper results in the absence of the transistorlike effect. More results of experimental study of the doped conducting-polymers based transistors can be found in recent publications. ${ }^{19-21}$

Because $I-V$ characteristics of the conducting polymerbased transistors are similar to that of semiconductor transistors, the first attempts ${ }^{20}$ to describe the observable field effect in the polymer were made by using the known models for conventional semiconductor field effect. However, it leads to unreasonable values of parameters ${ }^{20}$ and does not answer the main question of how the electric field penetrates in the polymer conductors with so high concentration of mobile charge carriers. According to Debye-Huckel theory, the electric field in such conductors should be screened on atomic or molecular scale. ${ }^{22,23}$ The screening length $r_{e}$ for a degenerate electron gas is given by the relation $r_{e}^{-2}=\left[e^{2} /\left(\varepsilon \varepsilon_{0}\right)\right] N\left(E_{F}\right)$, where $N\left(E_{F}\right)$ is the electron density of states at the Fermi level, $\varepsilon_{0}$ is the vacuum permittivity, and $\varepsilon$ is the relative dielectric constant of polymer. By using the constants $|e|=1.6 \times 10^{-19} \mathrm{C}$ and $\varepsilon_{0}=8.85 \times 10^{-12} \mathrm{C} /(\mathrm{m} \times \mathrm{V})$, the Debye radius is $r_{e}$ $=740 \varepsilon^{1 / 2} N\left(E_{F}\right)^{-1 / 2} \mathrm{~cm}^{-1 / 2}(\mathrm{eV})^{-1 / 2}$. The density of states at the Fermi level is estimated to be $N\left(E_{F}\right) \sim n_{e} / E_{F}$, where $n_{e}$ is the electron concentration. For a typical metal, $E_{F}$ is a few $\mathrm{eV}, n_{e} \sim 10^{22} \mathrm{~cm}^{-3}$, and $\varepsilon \sim 1$, the Debye radius $r_{e}$ is a few angstroms. In heavily doped conducting polymers, the typical charge-carrier concentration is estimated to be $n_{e} \sim b^{-3}$ $\sim 10^{21} \mathrm{~cm}^{-3}$ for typical in chain repeat constant $b \sim 1 \mathrm{~nm}$ and intrachain separation of 0.3 to $0.5 \mathrm{~nm}$. Taking the Fermi energy as $E_{F} \sim 1 \mathrm{eV}$, the density of states at the Fermi level is $N\left(E_{F}\right) \sim 10^{21} \mathrm{~cm}^{-3}(\mathrm{eV})^{-1}$, which is consistent with the value extracted from the Pauli-spin susceptibility. ${ }^{24}$ For the polymers with the characteristic dielectric constant $\varepsilon \sim 100,{ }^{25}$ the Debye radius then is $r_{e} \sim 2 \mathrm{~nm}$. The thickness of active polymer layer shown on Fig. 1 is $60 \mathrm{~nm}$ and, therefore, is much larger than the screening length.

Although the screening length is determined by solely the thermodynamic density of states, it is implicitly assumed that charge carriers are mobile. For conducting polymers near the IMT, a fraction of carriers are expected to be strongly localized and their mobility is very low. However, the mobility determines only how fast the screening happens. Irrespectively of transport mechanism, the characteristic screening time $t_{e}$ is given by the inverse conductivity as $t_{e} \sim \varepsilon \varepsilon_{0} / \sigma$. For the polymers with $\sigma_{\mathrm{RT}} \sim 10 \mathrm{~S} / \mathrm{cm}$ and $\varepsilon \sim 100$, the screening time should be very short $t_{e} \sim 10^{-11} \mathrm{~s}$.

Thus, the consideration of the conducting polymers as conventional conductors or semiconductors fails to explain the observable field effects. The experiments clearly show that the electric field deeply penetrates and changes the bulk conductivity of polymers. In Refs. 18 and 19, it was suggested that this happens due the above inhomogeneity of polymers and due to the presence of mobile ions in polymers. The inhomogeneous structure leaves enough free volume for migration of mobile ions. The mobile ions inside the 
polymers produce additional screening of the external field but the crucial feature of ions is its ability to migrate between the bulk polymer film and the external interface separating the film and a gate. As a result, the concentration of ions inside of the polymer is controlled by external electric field applied to the gate. Due to electroneutrality, the internal ionic density determines the concentration of primary charge carriers (holes) in the polymer and, thus, the polymer conductivity is governed by the external electric potential.

However, the recent experimental data ${ }^{21}$ find that the above electrochemical depletion of bulk charge produces small changes $(<2 \%)$ of the primary charge-carrier concentration and thereby itself cannot cause the observed large field effect. In Ref. 21, it was proposed that the field effect in conducting polymers is mainly due to the suppression of mobility of primary charge carriers (holes). Within the granular model, the mobility is determined by the hopping along the chains linking grains. The hopping sites on chains are associated with the interstitial counterion charges in amorphous regions. The introduction of a small amount of ions enables the suppression of intergrain hopping by removing hoping sites along chains by compensating the counterion charges with injected ion charges. Here, we present our model calculations and the experimental data that supports the present mechanism of field effect in the conducting polymers. In the beginning, for sake of completeness, we also consider in detail the model of conducting polymer-based electrochemical transistor. The model enables us to reproduce the $I(V)$ behavior, which is a characteristic for a transistor but does fail to fit the experimental dependencies.

The present article is organized in the following way. In Secs. III-V, starting with the drift-diffusion equation for electrons and ions experiencing a common self-consistent electrical potential, we derive the charge-voltage characteristics for an electrochemical capacitor and current-voltage characteristics of an electrochemical transistor. Then we review experimental data for the field effect in conducting polymers and conclude that the electrochemical mechanism of conductivity suppression is not adequate for conducting polymers. In Sec VI, we introduce the chain-linked granular model and derive the dependence of mobility as a function of ionic compensation. In Sec. VII, we summarize the obtained results.

\section{DRIFT-DIFFUSION EQUATIONS: GENERAL SCHEME}

To describe the phenomenon of field effect in conducting polymers, we use the drift-diffusion approximation for the charge current density of each type of charge carriers, holes or ions ${ }^{26}$

$$
J / z=-D \nabla n+\mu \mathrm{nF} .
$$

Here, $n=n\left(\zeta^{*}\right)$ is the equilibrium density of carriers with charge $z$ (holes or ions) determined by the corresponding local chemical potential $\zeta^{*}$ and $F$ is the electric field $F=-\nabla \varphi$ with $\varphi$ being an electric potential. The diffusion coefficient $D$ and the mobility $\mu$ in Eq. (1) are related by the Einstein equation

$$
z D\left(d n / d \zeta^{*}\right)=\mu \mathrm{n} .
$$

The relationship (2) enables representation of the partial current $[$ Eq. (1)] in the form

$$
J=-(\sigma / z) \nabla \zeta, \quad \zeta=\zeta^{*}+z \varphi, \quad \sigma\left(\zeta^{*}\right)=z^{2} D\left(d n / d \zeta^{*}\right),
$$

where the electrochemical potential $\zeta$ is introduced and $\sigma$ is the equilibrium conductivity. Equations. (1)-(3) assume that the interaction of charge carriers with the thermal bath is strong enough to provide the local equilibrium. ${ }^{25}$

The charge density $n$ in Eq. (3) or the corresponding local chemical potential $\zeta^{*}$ is determined from the charge conservation law

$$
z(\partial / \partial \mathrm{t}) n=-\nabla J .
$$

In Eq. (4), we ignore the charge exchange between primary charge carriers and ions. The electrical potential $\varphi$ in Eq. (3) is given by the Poisson equation

$$
-\varepsilon_{0} \nabla(\varepsilon \nabla \varphi)=\sum \mathrm{zn},
$$

where $\varepsilon$ is the relative permittivity of the sample. The right part of Eq. (5) includes the sum over all local charges.

Equations (4) and (5) require the explicit dependence of equilibrium density $n$ on the chemical potential $\zeta^{*}$. For ions the relation

$$
n_{i} / n_{i 0}=\exp \left[\zeta_{i}^{*} /\left(k_{B} T\right)\right], \quad n_{i 0}=n_{i}^{*} \exp \left[-E_{i} /\left(k_{B} T\right)\right],
$$

which is always valid provided that $n_{i} / n_{i}^{*} \ll 1$, where $n_{i}^{*}$ is the maximum affordable concentration of ions in the sample and $E_{i}$ is the corresponding standard electrochemical potential for ions.

The equilibrium electron density $n_{e}$ is given by

$$
n_{e}\left(\zeta_{e}^{*}\right)=\int N(E) f\left(E-\zeta_{e}^{*}\right) d E,
$$

where $f(E)$ is the Fermi-Dirac function

$$
f(E)=\left\{1+\exp \left[E /\left(k_{B} T\right)\right]\right\}^{-1} .
$$

In Eq. (7), $N(E)$ is the quantum-mechanical density of states for the primary charge-carrier energy band and

$$
\int N(E) d E \sim 1 / b^{3},
$$

where $b$ is the repeat constant for regular metals and $b$ includes the value of the intrachain and interchain repeat constants for polymer conductors.

For the degenerate electron gas (metals), $n_{e} \sim 1 / b^{3}$ and the electron chemical potential $\zeta_{e}^{*}$ are located deep $\left(\gg k_{B} T\right)$ inside the partially filled energy band so that one can write

$$
n_{e}\left(\zeta_{e}^{*}\right)=\int N(E) d E\left(E<\zeta_{e}^{*}\right), \quad d n_{e} / d \zeta_{e}^{*}=N\left(\zeta_{e}^{*}\right) .
$$

In the opposite case for the nondegenerate charge carriers (semiconductors), $n_{e} \ll 1 / b^{3}$, the chemical potential $\zeta_{e}^{*}$ is far $\left(\gg k_{B} T\right)$ outside the band and we have 
$n_{e}\left(\zeta_{e}^{*}\right)=n_{e 0} \exp \left[\zeta_{e}^{*} /\left(k_{B} T\right)\right], \quad n_{e 0}=\int N(E) \exp \left[-E /\left(k_{B} T\right)\right] d E$.

The set of Eqs. (4) and (5), with Eqs. (6), (8), and (9) supplied by the initial and boundary conditions, enables us to describe the operation of electrochemical devices. We consider a few of the applications of these equations.

\section{SCREENING}

We assume that the external electrical field $F$ is applied to a conducting sample. Inside the sample there is the electric potential $\varphi$ so that near the sample surface, $\left.\nabla \varphi\right|_{\Gamma}=-F / \varepsilon$, but any current is absent at the surface. Then from the equation $J_{e}=0$, it follows that $\nabla \zeta_{e}=0$ or $\zeta_{e}=\zeta_{e}^{*}+z \varphi$ is constant, and the Poisson Eq. (5) for the electric potential $\varphi$ in the absence of ions reads

$$
\varepsilon_{0} \varepsilon \Delta \varphi=-z_{e}\left[n_{e}(\zeta-z \varphi)-n_{e}(\zeta)\right] .
$$

In a metallic sample, $\left[n_{e}(\zeta-z \varphi)-n_{e}(\zeta)\right]=-N\left(E_{F}\right) z \varphi$ and

$$
\varphi=\left(F r_{e} / \varepsilon\right) \exp \left[-x / r_{e}\right], \quad \varepsilon_{0} \varepsilon / r_{e}^{2}=z_{e}^{2} N\left(E_{F}\right),
$$

where $\zeta^{*}=E_{F} \gg k_{B} T$ is the Fermi energy. As we discussed in Sec. I, the screening length in metals is of the lattice constant. Therefore, the chemical potential turns constant inside the metal.

For the description of charge screening in semiconductors, we use the identity $\left[n_{e}(\zeta-z \varphi)-n_{e}(\zeta)\right]=n_{e}(\zeta)$ $\times\left[\exp \left(-z \varphi / k_{B} T\right)-1\right]$, which enables us to rewrite the Poisson equation in the form

$$
\varepsilon_{0} \varepsilon \Delta \varphi=-z_{e}\left[\exp \left(-z \varphi / k_{B} T\right)-1\right] .
$$

Using the other identity,

$$
d^{2} \varphi / d x^{2}=(1 / 2)(d / d \varphi)(d x / d \varphi)^{-2},
$$

and introducing variables

$$
v=z_{e} \varphi /\left(k_{B} T\right), \quad y=x / r_{e}, \quad \varepsilon_{0} \varepsilon / r_{e}^{2}=z_{e}^{2} n_{e} /\left(k_{B} T\right),
$$

from Eq. (12) we get

$$
y=2_{v 0}^{-1 / 2} \int^{v} d v[v+\exp (-v)-1]^{-1 / 2} .
$$

According to Eq. (15), far from the boundary $y \gg 1$, the potential $v$ decays the same way as in a metal, Eq. (11), i.e.,

$$
v \sim \exp (-y) \text {. }
$$

Equation (16) proves that the expression for Debye radius

$$
1 / r^{2}\left(\zeta^{*}\right)=\left(z^{2} / \varepsilon \varepsilon_{0}\right) d n\left(\zeta^{*}\right) / d \zeta^{*},
$$

which initially is derived for a weak field, is valid also for any electric field. Universality of Eq. (17) is a consequence of screening that makes any electric field eventually weak.

Including in the consideration of ion charges provides additional screening of an electric field and the total Debye radius, $r_{D}$ becomes

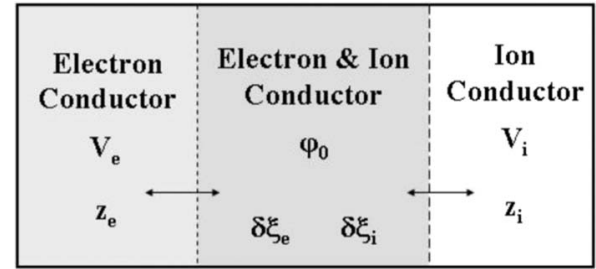

FIG. 2. Schematic of electrochemical capacitor.

$$
1 / r_{D}^{2}=1 / r_{e}^{2}+1 / r_{i}^{2},
$$

where $r_{i, e}$ is the screening length provided by ions and electrons [Eq. (17)].

\section{CHARGING}

The description of an electrochemical device is simplified if its size is much larger than any screening length. As a next example, we consider the electrochemical capacitor (supercapacitor) ${ }^{27}$ where electrical potential is applied between the conducting polymer and gate separated from the polymer by electrolyte (an ion conductor) as it is shown in Fig. 2. The electrical potential of the electrolyte is assumed to follow that of the gate (electrochemical gating) provided there is the intensive redox exchange between the electrolyte and the gate. The absence of a partial charge current through an interface means that the corresponding electrochemical potential is constant across the given interface. Therefore, the following boundary relations hold

$$
\delta \zeta_{i}^{*}+z_{i} \delta \varphi=z_{i} V_{i}, \quad \delta \zeta_{e}^{*}+z_{e} \delta \varphi=z_{e} V_{e},
$$

where $\delta \zeta_{i, e}$ and $\delta \varphi$ are the changes of chemical and electrical potentials inside the film due to the external electrical potentials applied to the electrolyte (ionic contact) $V_{i}$ and the polymer (electronic contact) $V_{e}$. From Eq. (19), it follows that

$$
\delta \varphi=V_{i}-\delta \zeta_{i}^{*} / z_{i}=V_{e}-\delta \zeta_{e}^{*} / z_{e}, \quad \delta \zeta_{i}^{*} / z_{i}-\delta \zeta_{e}^{*} / z_{e}=V_{i}-V_{e} .
$$

The requirement of the electroneutrality, which now replaces the Poisson equation, should be added for Eq. (20) to get

$$
z_{i} \delta n_{i}+z_{e} \delta n_{e}=0 .
$$

In the linear-response regime, $\delta n_{i}=\left(\varepsilon \varepsilon_{0} / r_{i}^{2}\right)\left(\delta \zeta_{i}^{*} / z_{i}^{2}\right)$ and $\delta n_{e}=\left(\varepsilon \varepsilon_{0} / r_{e}^{2}\right)\left(\delta \zeta_{e}^{*} / z_{e}^{2}\right)$, and then from Eqs. (20) and (21), it follows

$$
\delta \varphi=\left(V_{i} r_{e}^{2}+V_{e} r_{i}^{2}\right) /\left(r_{i}^{2}+r_{e}^{2}\right), \quad \delta \zeta_{i}^{*} / z_{i}=\left(V_{i}-V_{e}\right) r_{i}^{2} /\left(r_{i}^{2}+r_{e}^{2}\right) .
$$

We define the differential capacity, $c$, per unit volume by the following the equation

$$
\begin{gathered}
q=z_{i} \delta n_{i}=-z_{e} \delta n_{e}=\left(V_{i}-V_{e}\right) \varepsilon \varepsilon_{0} / r_{c}^{2}=c\left(V_{i}-V_{e}\right), \\
c=\varepsilon \varepsilon_{0} / r_{c}^{2}, \quad r_{c}^{2}=r_{i}^{2}+r_{e}^{2} .
\end{gathered}
$$

Thus, according to Eq. (24), the total capacity, $c$, is given by series of partial capacities of its constituents, 


$$
1 / c=1 / c_{i}+1 / c_{e}, \quad 1 / c_{\alpha}=\varepsilon \varepsilon_{0} / r_{\alpha}^{2}=z_{\alpha}^{2} d n_{\alpha}\left(\zeta_{\alpha}^{*}\right) / d \zeta_{\alpha}^{*}, \quad \alpha=i, e .
$$

This relationship for linear response of polymers was derived earlier in Refs. 28 and 29.

Equation (25) is directly generalized beyond the linear regime. For some particular cases, it is possible to get explicit dependence of specific capacity, $c$, on the applied voltage. For a nondegenerate electron gas described by Eq. (9) and for $z_{e}=-z_{i}$ from Eqs. (20) and (21), one can find

$$
n_{e}=n_{e 0} p, \quad n_{i}=n_{i 0}+n_{e 0}(p-1),
$$

where

$$
\begin{gathered}
p=(1-a) / 2+\left[(1-a)^{2} / 4+a \exp (v)\right]^{1 / 2}, \\
a=n_{i 0} / n_{e 0}, \quad v=z_{i}\left(V_{i}-V_{e}\right) /\left(k_{B} T\right) .
\end{gathered}
$$

For $z_{e}=z_{i}$, the electronic and ionic densities vary with applied voltage as

$$
\begin{gathered}
n_{e}=n_{e 0}(a+1) /[a \exp (v)+1] ; \\
n_{i}=n_{i 0}(a+1) \exp (v) /[a \exp (v)+1] .
\end{gathered}
$$

For degenerate electrons, we assume that the density of states near the Fermi level, $N\left(E_{F}+\delta \zeta_{e}^{*}\right)$, remains constant, at least, as long as $\left|\delta \zeta_{e}^{*}\right| \ll E_{F}$. The corresponding screening length $r_{e}$ is given by the equation

$$
\varepsilon \varepsilon_{0} / r_{e}^{2}=z_{e}^{2} N\left(E_{F}\right)
$$

Introducing the variables

$$
1 / a=\left(z_{e} / z_{i}\right)^{2} N\left(E_{F}\right) k_{B} T / n_{i 0}=\left(r_{i} / r_{e}\right)^{2}, \quad x=\left(z_{i} / z_{e}\right) \delta \zeta_{e}^{*} /\left(k_{B} T\right),
$$

Eq. (21) for the determination of equilibrium electron and ion concentrations reads

$$
1-x / a=\exp (v+x)
$$

Defining, the capacitance density $c$ as $q=z_{i} \delta n_{i}=-z_{e} \delta n_{e}=\mathrm{cV}$ and $c_{0}=\varepsilon \varepsilon_{0} / r_{c}^{2}$, we have

$$
c / c_{0}=-(1+1 / a)(x / v),
$$

where $x$ is found from Eq. (31). From Eqs. (31) and (32), it follows that the linear regime $c=c_{0}$ holds as long as $|v| /(1$ $+a) \ll 1$ and $x=-a v /(1+a)$.

For $a \gg 1\left(r_{i} \ll r_{e}\right)$, the capacity $c$ from Eq. (32) remains always constant $\left(c_{0}\right)$ as long as electron chemical potential (the Fermi level) does not reach the band edges and the assumption about the degenerate electron gas fails. Taking into account that $N\left(E_{F}\right) \sim n_{e}^{0} / E_{F}$ and the typical electron concentration in heavily doped polymers is $n_{e 0} \sim 10^{21} \mathrm{~cm}^{-3}$ and for the Fermi energy $E_{F} \sim 1 \mathrm{eV}$, the density of states at the Fermi level is $N\left(E_{F}\right) \sim 10^{21} \mathrm{~cm}^{-3}(\mathrm{eV})^{-1}$. Plugging $z_{e}=|e|$ $=1.6 \times 10^{-19} C=1.6 \times 10^{-19} \mathrm{~F} \times \mathrm{V}$, we find that in this case $(a \gg 1)$, the capacity $c_{0}$ is around $100 \mathrm{~F} / \mathrm{cm}^{3}$. The condition $a \gg 1$ is satisfied for the concentration of ions $n_{i 0}$ $>n_{e 0}\left(k_{B} T / E_{F}\right) \sim 3 \times 10^{19} \mathrm{~cm}^{-3}$ at $T=300 \mathrm{~K}$.

At $a \ll 1 \quad\left(r_{i} \gg r_{e}\right.$ or $\left.n_{i 0}<n_{e 0}\left(k_{B} T / E_{F}\right) \sim 3 \times 10^{19} \mathrm{~cm}^{-3}\right)$, the nonlinear regime is realized. As long as $v \ll \ln (1 / a)$, the capacity $c$ and ionic concentration exponentially increases

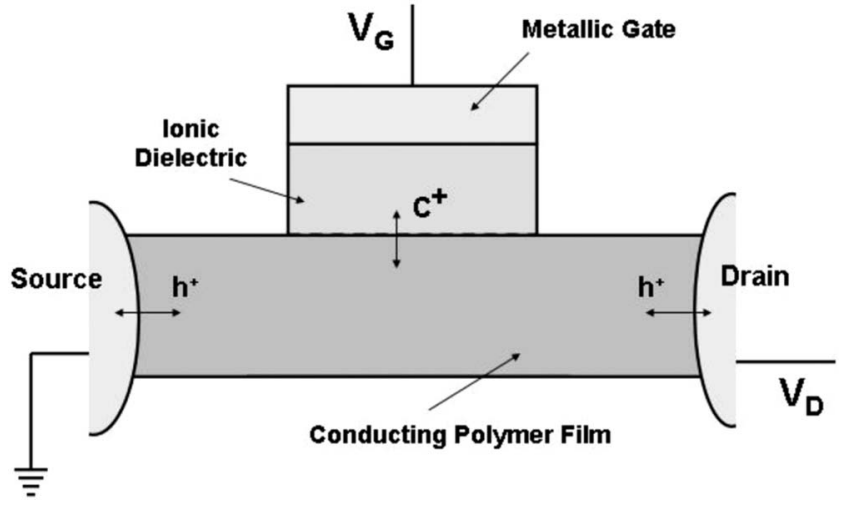

FIG. 3. Schematic of electrochemical transistor.

with the applied voltage, $c / c_{0}=[\exp (v)-1] / v$, where $c_{0}$ $=\varepsilon \varepsilon_{0} / r_{i}^{2}=z_{i}^{2} c_{i}^{0} /\left(k_{B} T\right)$. The capacity $c$ approaches the saturation $c=c_{0} / a=\varepsilon \varepsilon_{0} / r_{e}^{2}=z_{e}^{2} N /\left(E_{F}\right) \sim z_{e}^{2} n_{e}^{0} / E_{F}, \quad$ as $c=[1$ $-(1 / v) \ln (v / a)] c_{0} / a$ at $v \gg \ln (1 / a)$.

It should be stressed that here the voltage is applied between the ionic and electronic contacts each of which has only one type of charge carrier (see Fig. 2). The electrochemical sample is assumed to have both types of charge carriers.

\section{GATING}

Finally, we consider the field effect in the conducting polymers. The device geometry is shown on Fig. 3 and differs from the electrochemical capacitor discussed in Sec. IV by the presence of two separated electronic contacts: source and drain. In the stationary regime, any ionic current is absent and a gate potential $V_{g}$ is screened by holes, as a result, the electrochemical potential for ions inside the polymer film becomes $\zeta_{i}=\zeta_{i} V_{g}$ and hence the ionic chemical potential reads $\zeta_{i}^{*}=z_{i}\left(V_{g}-\varphi\right)$, where $\varphi$ is the spatially varying electric potential inside the film. In the beginning, we consider the nondegenerate case when the concentrations of electrons and ions as a function of the corresponding chemical potential are described by Eqs. (6) and (9). We assume also that $z_{i}$ $=z_{e}|e|$, i.e., we deal with holes and cations as it take place in the experiments. ${ }^{17-21}$

From the requirement of electroneutrality [Eq. (21)], it follows that $\varphi$ satisfies the equation

$$
\begin{aligned}
\exp \left[-z_{e} \varphi / k_{B} T\right]= & \left(n_{e 0}+n_{i 0}\right) /\left[n_{e 0} \exp \left(\zeta_{e} / k_{B} T\right)\right] \\
& +n_{i 0} \exp \left(z_{e} V_{g} / k_{B} T\right) .
\end{aligned}
$$

The electric potential $\varphi$ also should satisfy the equation $d^{2} \varphi / d x^{2}=0$, and at the end, we check that indeed this equality holds. The expression for hole current [Eq. (3)] with Eq. (33) now reads

$$
\begin{aligned}
J_{e}= & -z_{e} D_{e}\left(n_{e 0}+n_{i 0}\right)(d / d x) \ln \left[n_{e 0} \exp \left(\zeta_{e} / k_{B} T\right)\right. \\
& \left.+n_{i 0} \exp \left(z_{e} V_{g} / k_{B} T\right)\right],
\end{aligned}
$$

where $D_{e}$ is the diffusion coefficient for holes. In the stationary regime, the requirement $J_{e}=-I$ (I-const. is the measured current in the external circuit) determines the electrochemical potential $\zeta_{e}$ as a function of $x$ 


$$
\begin{aligned}
n_{e 0} \exp \left(\zeta_{e} / k_{B} T\right)+n_{i 0} \exp \left(z_{e} V_{g} / k_{B} T\right) \\
=\left[n_{e 0}+n_{i 0} \exp \left(z_{e} V_{g} / k_{B} T\right)\right] \\
\quad \times \exp \left\{I x /\left[z_{e} D_{e}\left(n_{e 0}+n_{i 0}\right)\right]\right\} .
\end{aligned}
$$

We assume here that the source contact is grounded, i.e., $\zeta_{e}(0)=0$.

In this way according to Eqs. (33) and (34), the electrical potential $\varphi$ is given by the equation

$$
\begin{aligned}
\exp \left(z_{e} \varphi / k_{B} T\right)= & \left(n_{e 0}+n_{i 0}\right)^{-1}\left[n_{e 0}+n_{i 0} \exp \left(z_{e} V_{g} / k_{B} T\right)\right] \\
& \times \exp \left\{I x /\left[z_{e} D_{e}\left(n_{e 0}+n_{i 0}\right)\right]\right\} .
\end{aligned}
$$

The electric potential from Eq. (35) provides the requirement that the electric field $F=-\nabla \varphi$ is constant and $d^{2} \varphi / d x^{2}=0$ inside the film. Also, the above solution [Eq. (34) and (35)] provides $\zeta_{i}=z_{i} V_{g}$-const. and, therefore, there is no ionic current in the active channel.

Defining the voltage drop across the sample as $\zeta_{e}(L)$ $=V_{D}$, where $L$ is the length of channel, from Eq. (35), we find $I(V)$ characteristics of the device

$$
I\left(V_{D}, V_{G}\right)=I_{0}(1+a) \ln \left[\left(e^{u}+a e^{v}\right) /\left(1+a e^{v}\right)\right],
$$

where we introduce the new variables

$$
v=z_{i} V_{G} /\left(k_{B} T\right), \quad u=z_{i} V_{D} /\left(k_{B} T\right),
$$

and the parameters

$$
\begin{gathered}
a=n_{i 0} / n_{e 0} ; \quad I_{0}=\left(1 / R_{0}\right)\left(k_{B} T / z_{i}\right) ; \\
R_{0}=L /\left(S \sigma_{0}\right) ; \quad \sigma_{e 0}=z_{e}^{2} D_{e} n_{e 0} /\left(k_{B} T\right),
\end{gathered}
$$

with $S$ being the cross section of conducting channel and $R_{0}$ is the floating device resistance in a linear regime.

$I(V)$ characteristics given by Eq. (36) demonstrate the behavior which is typical for conventional transistors (see Fig. 4). In the linear limit along the drain voltage, the conductance of the device is

$$
G(v)=\left(1 / R_{0}\right)(1+a) /[1+a \exp (v)] .
$$

We can define the cutoff gate voltage $v_{c}$, when $G\left(v_{c}\right)$ $\ll 1 / R_{0}$. For the case of our interest $a \ll 1$ from Eq. (39) we have

$$
v_{c}=-\ln (a) .
$$

The $I(V)$ from Eq. (36) also demonstrates the regime of "plateau" at the negative drain voltage $u<u_{s}=v-v_{c}<0$ with the saturation current, $I_{s}$, given by the equation

$$
I_{s}=-I_{0}(1+a) \ln \left[1+\exp \left(-u_{s}\right)\right] .
$$

The mechanism leading to the plateau regime is similar to that for conventional semiconductor transistors. Increasing the drain voltage increases the driving field inside the film but simultaneously suppresses the conductivity by decreasing the charge-carrier density. According to Eqs. (34) and (35), the distribution of electron density along the active channel is
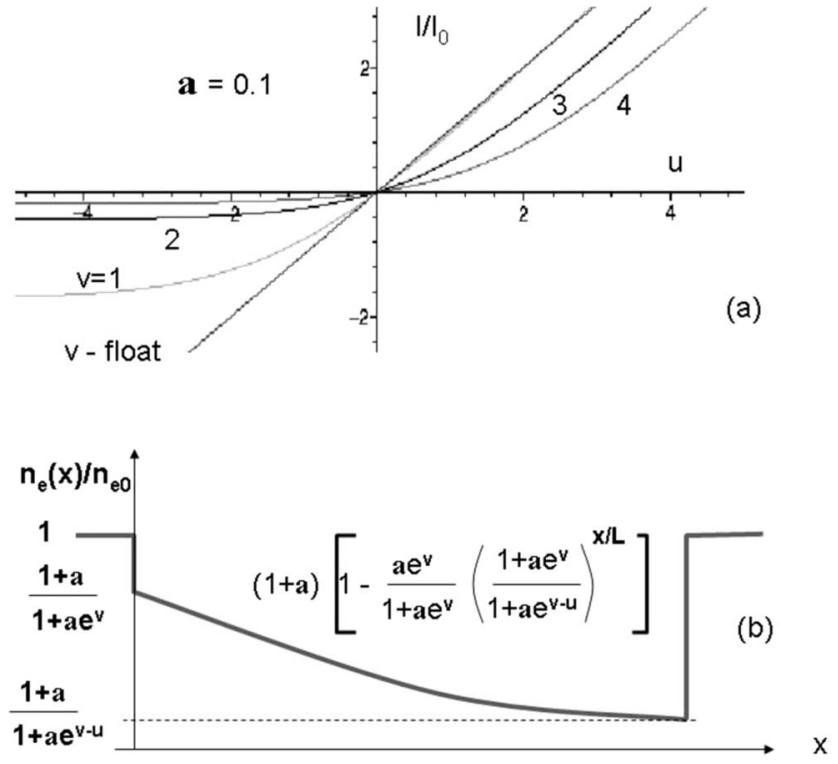

FIG. 4. (a) Predicted $I(V)$ characteristics of electrochemical transistor for different gate voltages. (b) Schematic distribution of electron charge inside the electrochemical transistor active channel. Here, $a=n_{0 i} / n_{0 e}$ is the initial ratio of ionic to electronic densities; $u=e U_{\mathrm{SD}} / k_{B} T ; v=\mathrm{eV}_{G} / k_{B} T$.

$$
\begin{aligned}
n_{e}(x) / n_{e 0}= & (1+a)\left\{1-a e^{v}\left(1+a e^{v}\right)^{-1}\right. \\
& \left.\times\left[\left(1+a e^{v}\right) /\left(e^{u}+a e^{v}\right)\right]^{x / L}\right\},
\end{aligned}
$$

and varies from $n_{e 0}(1+a) /\left(1+a e^{v}\right)$ at $x=0$ to $n_{e 0}(1+a) /(1$ $+a e^{v-u}$ ) for $x=L$ (see Fig. 4).

Above, we consider a nondegenerate electron gas. Within the model of a degenerate electron gas, the conductivity is determined by only the density of states and the diffusion coefficient for holes at the Fermi level. Therefore, the conductivity does change with the gate voltage so long as the electron chemical potential (the Fermi level) does not approach the bottom of conduction band. At those gate voltages, the hole concentration becomes small so that the description derived for nondegenerate electron gas becomes adequate. Therefore, we anticipate that the above $I(V)$ characteristics, given by Eq. (36), are universal for electrochemical transistors at least at a qualitative level.

\section{ION CONTROL OF ELECTRON MOBILITY}

It is natural to anticipate for the heavily doped conducting polymers that the concentration of remnant mobile ions is small in comparison with the primary charge density, i.e., the parameter $a \ll 1$. In this case, the bulk capacity of the polymer is determined by the ion component and the changes of hole concentration and, hence, the changes of hole conductivity demonstrates the sharp exponential dependence with the gate voltage. The experimental dependence is rather smooth with the gate voltage. ${ }^{17-21}$ For this reason, we failed to obtain satisfactory fit of the experimental $I(V)$ characteristics using the derived theoretical dependencies, Eqs. (36)-(39). 
Similar to the model of conventional semiconductor transistor $^{30}$ above, we assume the constant mobility for primary charge carriers (holes). Therefore, the change of device conductance is solely due to the change of the number of charge carriers. As a result, the model predicts a substantial change of conductivity if only a significant fraction of primary charge carriers is replaced with ions. The experimental data ${ }^{19,21}$ does not support this conclusion. The critical degree of charge compensation, the ratio of injected ionic charge to the total hole charge at which the conductivity drops by an order of magnitude, is of only a few percent $(2 \%-5 \%) .{ }^{21}$ Therefore, the decrease of hole concentration itself is not the factor which determines the observed field effect.

We propose that, simultaneous with the variation of charge-carrier concentration, there is the noticeable variation of mobility of charge carriers which accounts for the observed field effect in the conducting polymer. The sharp increase of mobility by a few orders of magnitude with increase of charge concentration in the process of doping conjugated polymers was reported in a number of works. ${ }^{31-33}$ The increase is explained by a fact that at low doping, the charge carriers are trapped by Coulomb forces near dopants or deep traps. ${ }^{32,33}$ With increase of doping, the Coulomb interaction is screened, the deep traps are filled, and afterwards the charge carriers are released for charge transport.

In this section, we describe the field effect in conducting polymers by following a similar approach. However, it should be stressed that for conducting polymers, the large change of mobility with small variation of charge concentration occurs in the regime of heavy doping. ${ }^{19,21}$ Therefore, the mobility cannot be controlled by traps as trapping takes place at light doping. We propose that the field effect in conducting polymers is an electric-field induced conductornonconductor transition described by the chain-linked granular model. ${ }^{18,19,21}$ Within the present model, the charge transport is controlled by intergrain hopping of holes through intermediate states in the disordered regions separating grains. The hopping states that form the intergrain bridges for holes are associated with localized states on polymer chains near to acceptors (e.g., PSS $^{-}$). These acceptors sitting in a disordered less dense regions are easy accessible for interaction with the injected cations, and their charges are first to be compensated during doping (Fig. 5). The compensation of acceptor charges as injected cations removes the intergrain hopping states for holes, and the electronic states at the Fermi level become more localized resulting in the decrease of conductivity.

To obtain a more quantity comparison, we consider the temperature dependence of typical devices. According to the chain-linked granular model, the temperature dependence of resistivity is given by the quasi-one-dimensional Mott's law: ${ }^{13,15}$

$$
R_{f}(T)=R_{0} \exp \left[\left(T_{1} / T\right)^{1 / 2}\right],
$$

where the temperature dependence scale $T_{1}$ in Eq. (42) is determined by characteristics of electronic states of single chains
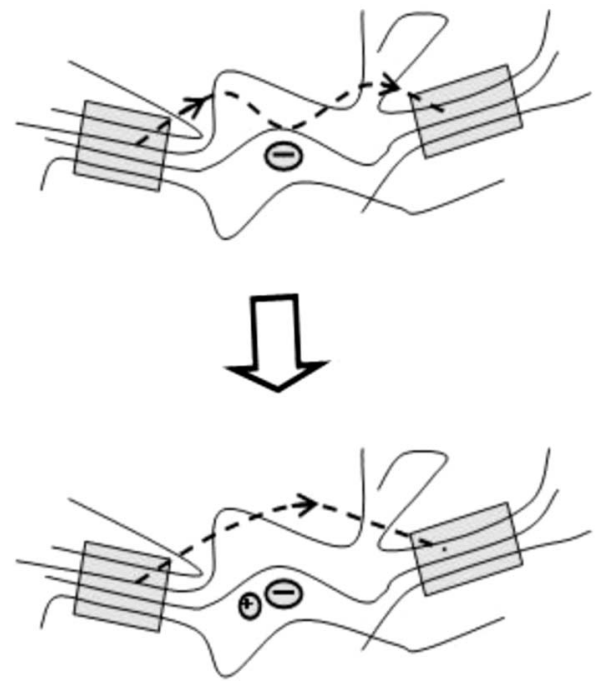

FIG. 5. Ionic suppression of intergrain hopping. The compensation by cations of negative charge of acceptors removes the nearby localized states that provides for holes easy hopping between grains.

$$
T_{1}=b /\left[N_{1} \xi\right]
$$

Here, $N_{1}$ is the one-dimensional (1D) Fermi density of states, $\xi$ is the localization length along a chain and $b$ is the numerical factor $\left(b=16,{ }^{25}\right)$. As we already discussed, the hopping states along chains linking the nearest grains are associated with negatively charged acceptors in strongly disordered regions. Therefore, the parameter $N_{1}$ in Eq. (43) can be approximated as $N_{1} \sim n_{d} / \mathrm{W}$, where $n_{d}$ is the linear concentration of acceptors and $W$ is the characteristic energy-level spread. Injected cations compensate acceptor charges and a number of available hopping states along the polymer backbone decreases. Then $T_{1}$ as a function of compensation degree, $q$, increases as

$$
T_{0} / T_{1}=1-\alpha q,
$$

with $\alpha$ is the material dependent constant.

The temperature dependence of the conductivity of the active channel at different levels of ion compensation was studied in Refs. 19 and 21. Cations are injected at room temperature by applying a positive gate voltage. As it was shown in Ref. 21, the increase of channel resistance $R_{f} / R_{0}$, where $R_{0}$ is the initial resistance at $300 \mathrm{~K}$, and $R_{f}$ is the room-temperature resistance at the applied gate voltage, which actually depends on the total injection charge or the level compensation $q$. In Fig. 6, the resistance of the conducting polymer-based transistor as a function of temperature is shown for the different $R_{f} / R_{0}$ or the different level of compensation.

The experimental data on Fig. 6 can be fit to the quasione-dimensional Mott's law [Eq. (43)], where the temperature scale $T_{1}$ varies with the applied voltage. It enables us to establish the dependence of $T_{1}$ on the applied gate voltage and on the degree of compensation. The inset on Fig. 6 demonstrates the experimental dependence of $T_{1}$ on compensa- 


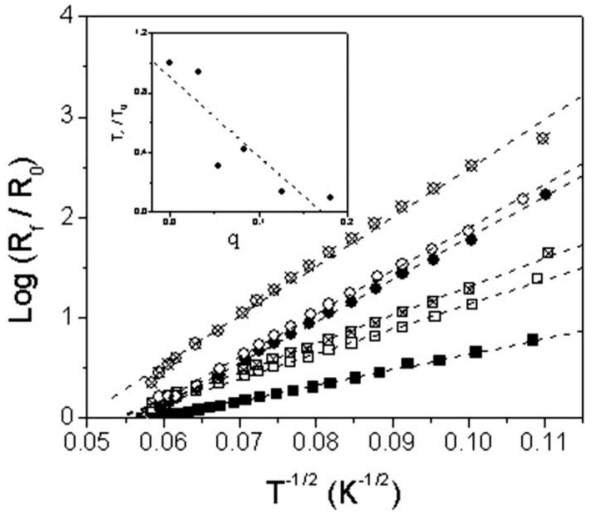

FIG. 6. Quasi-one-dimensional Mott's law fit for temperature dependent resistance at different room-temperature conducting states $R_{f} / R_{0}$ induced by the different applied gate voltage: $R_{f}(300 \mathrm{~K}) / R_{0}(300 \mathrm{~K})=1(), 2.2(\square), \quad 7.8(\otimes), \quad 15.6(\bullet), 35.7(\bigcirc)$ and $100(\otimes)$. Inset: Characteristic temperature scale of quasi-onedimensional Mott's law vs the compensation level (ion charge/hole charge) [after Hsu et al. (Ref. 21)].

tion level $q$. Linear fitting of the dependence $T_{1}(q)$ in accordance with Eq. (44) gives slope coefficient as $\alpha \sim 7$ and the critical compensation as a few percent.

We can estimate the critical concentration of ions within the present model based on the following simple argument. If we assume $50 \%$ crystallinity and the size grain $\sim 10 \mathrm{~nm}$, then intergrain hopping includes $\sim 10$ intermediate sites along the chain link. To interrupt this connection, it is enough to introduce only one ion, i.e., approximately $5 \%$ "dedoping" may produce an appreciable effect.

\section{CONCLUSION}

The undoped conjugated polymers are semiconductors and are now often used as active elements in field effect transistors. ${ }^{34}$ The operation of those devices is described within the canonical model of field effect transistor developed for crystalline semiconductors. ${ }^{30}$ They work often in the accumulation regime which is provided by bending of the band edge with the gate voltage near the interface. The charge carriers from the bulk of the semiconductor occupy these new states and increase the conductivity of the active channel. For the case of thin organic layers, the theory recently was adjusted by Stallinga and Gomes. ${ }^{35}$ All these models assume the constant charge-carrier mobility and that the change of conductivity is due to variation of charge concentration with gate voltage. In real devices, there is a dependence of mobility on the gate voltage or on the charge concentration and this dependence occurs to be strong at low charge concentration when the filling of trap states takes place.
The present work addressed the field effect transistors based on heavily doped conjugated polymers. These polymers are no longer semiconductors but metals with conductivity $\sim 10 \mathrm{~S} / \mathrm{cm}$ and charge-carrier density $\sim 10^{21} \mathrm{~cm}^{-3}$. Surprisingly, such transistors demonstrate IV characteristics which are similar to those of regular transistors but they work on a different principle.

It was suggested that the field effect in conducting polymer-based transistor is related to the insertion of small density of ions into conducting polymer. ${ }^{19,21}$ The porous structure of the polymer leaves space for electrolyte. Then the ionic chemical potential inside of the polymer is controlled by the gate potential. Due to electroneutrality, the electrochemical potential for ions and electrochemical potential for electrons (Fermi level) are interdependent. In such a way, the concentration of electrons is governed by the gate potential (electrochemical gating). In contrast to the regular transistor in the conducting polymer-based transistor, the charge density of the active channel varies not only near the gate electrode interface but across the whole active channel thickness.

In the present work, we have shown that such a simple model does not enable us to quantitively describe the observable field effect in conducting polymer-based transistors. The experiments ${ }^{19,21}$ have shown that the small fraction of ions to the total number of charge carriers $(\sim 2 \%)$ enables to suppress the conductivity by a factor $10 .{ }^{21} \mathrm{We}$ have shown here that these strong changes of conductivity are rather related to the strong changes of mobility of holes due to morphology of the conducting polymers.

We suggest that the field effect in conducting polymers is an inherent feature of the inhomogeneous structure of polymeric networks including the existence of dense and well ordered crystalline regions. The crystalline domains can be considered like metallic grains or dots of mesoscopic size embedded in porous and poorly conducting media. The main effect of applied positive gate voltage is the suppression of the intergrain hopping by partial charge compensation (dedoping) of the disordered polymer media. As a result, the conductor-nonconductor transition occurs. Because the conducting network is close to the percolation threshold, a small decrease (compensation) of hopping sites is able to produce such a transition.

This conclusion is consistent with an early electrochemical study which shows that it is difficult to get a complete dedoping of highly conducting polymers due to doped crystalline domains remaining inside the polymers. ${ }^{36,37}$

\section{ACKNOWLEDGMENTS}

The present work in part supported by DOE and AFOSR. 
${ }^{1}$ A. J. Heeger, Angew. Chem., Int. Ed. 40, 2591 (2001); A. G. MacDiarmid, ibid. 40, 2581 (2001); H. Shirakawa, ibid. 40, 2575 (2001).

${ }^{2}$ A. J. Heeger, S. Kivelson, J. R. Schriffer, and W. P. Su, Rev. Mod. Phys. 60, 781 (1988).

${ }^{3}$ E. J. Mele and M. J. Rice, Phys. Rev. B 23, 5397 (1981).

${ }^{4}$ S. A. Kivelson and A. J. Heeger, Phys. Rev. Lett. 55, 308 (1985).

${ }^{5}$ C. K. Chiang, C. R. Fincher, Y. W. Park, A. J. Heeger, H. Shirakawa, E. J. Louis, S. C. Gau, and A. G. MacDiarmid, Phys. Rev. Lett. 39, 1098 (1977).

${ }^{6}$ A. J. Epstein, J. Joo, R. S. Kohlman, G. Du, E. J. Oh, Y. Min, J. Tsukamoto, H. Kaneko, and J. P. Pouget, Synth. Met. 65, 149 (1994).

${ }^{7}$ N. F. Mott and E. Davis, Electronic Processes in Non-Crystalline Materials (Clarendon, Oxford, 1979); B. I. Shklovskii and A. L. Efros, Electronic Properties of Doped Semiconductors (Springer-Verlag, Berlin, 1984).

${ }^{8}$ P. A. Lee and T. R. Ramakrishnan, Rev. Mod. Phys. 57, 287 (1985).

${ }^{9}$ A. N. Samukhin, V. N. Prigodin, L. Jastrabik, and A. J. Epstein, Phys. Rev. B 58, 11354 (1998).

${ }^{10}$ J. A. Reedijk, H. C. F. Martens, H. B. Brom, and M. A. J. Michels, Phys. Rev. Lett. 83, 3904 (1999); A. J. Epstein, W.-P. Lee, and V. N. Prigodin, Synth. Met. 117, 9 (2001); V. N. Prigodin and A. J. Epstein, ibid. 141, 155 (2004).

${ }^{11}$ T. Ishiguro, H. Kaneko, Y. Nogami, H. Ishimoto, H. Nishiyama, J. Tsukamoto, A. Takahashi, M. Yamaura, T. Hagiwara, and K. Sato, Phys. Rev. Lett. 69, 660 (1992); R. S. Kohlman, J. Joo, Y. G. Min, A. G. MacDiarmid, and A. J. Epstein, ibid. 77, 2766 (1996); R. S. Kohlman, A. Zibold, D. B. Tanner, G. G. Ihas, T. Ishiguro, Y. G. Min, A. G. MacDiarmid, and A. J. Epstein, ibid. 78, 3915 (1997).

${ }^{12}$ R. S. Kohlman and A. J. Epstein, in Handbook of Conducting Polymers, 2nd ed. edited by T. Skotheim, R. Elsenbaumer, and J. Reynolds (Dekker, New York, 1998), pp. 85-121; A. J. Epstein, Organic Electronic Materials: Conjugated Polymers and Low Molecular Weight Organic Solids, Springer Series in Materials Science Vol. 41, edited by R. Farchioni and G. Grosso (SpringerVerlag, Berlin, 2001), pp. 3-39; A. J. Epstein, J. Joo, R. S. Kohlman, G. Du, A. G. MacDiarmid, E. J. Oh, Y. Min, J. Tsukamoto, H. Kaneko, and J. P. Pouget, Synth. Met. 65, 149 (1994); J. Joo, Z. Oblakowski, G. Du, J. P. Pouget, E. J. Oh, J. M. Wiesinger, Y. Min, A. G. MacDiarmid, and A. J. Epstein, Phys. Rev. B 49, 2977 (1994); J. Joo, V. N. Prigodin, Y. G. Min, A. G. MacDiarmid, and A. J. Epstein, ibid. 50, 12226 (1994).

${ }^{13}$ R. Menon, C. O. Yoon, D. Moses, and A. J. Heeger, in Handbook of Conducting Polymers, 2nd ed., edited by T. Skotheim, R. Elsenbaumer, and J. Reynolds (Dekker, New York, 1998), pp. 27-84.

${ }^{14}$ H. C. F. Martens, J. A. Reedijk, H. B. Brom, D. M. deLeeuw, and R. Menon, Phys. Rev. B 63, 073203 (2001).

${ }^{15}$ V. N. Prigodin and A. J. Epstein, Synth. Met. 125, 43 (2001); V.
N. Prigodin and A. J. Epstein, Europhys. Lett. 60, 750 (2002).

${ }^{16}$ J. P. Pouget, Z. Oblakowski, Y. Nogami, P. A. Albouy, M. Laridjani, E. J. Oh, Y. Min, A. G. MacDiarmid, J. Tsukamoto, T. Ishiguro, and A. J. Epstein, Synth. Met. 65, 131 (1994); M. Laridjani and A. J. Epstein, Eur. Phys. J. B 7, 585 (1999); J. P. Travers, B. Sixou, D. Berner, A. Wolter, P. Rannou, B. Beau, B. Pepen-Donat, C. Barther, M. Guglielmi, N. Mermolliod, B. Gilles, D. Djurado, A. J. Attias, and M. Vautrin, Synth. Met. 101, 359 (1999).

${ }^{17}$ J. Lu, N. J. Pinto, and A. G. MacDiarmid, J. Appl. Phys. 92, 6033 (2002).

${ }^{18}$ A. J. Epstein, F.-C. Hsu, N.-R. Chiou, and V. N. Prigodin, Curr. Appl. Phys. 2, 339 (2002).

${ }^{19}$ A. J. Epstein, F.-C. Hsu, N.-R. Chiou, and V. N. Prigodin, Synth. Met. 137, 859 (2003)

${ }^{20}$ H. Okuzaki, M. Ishihara, and S. Ashizawa, Synth. Met. 137, 947 (2003).

${ }^{21}$ F. C. Hsu, V. N. Prigodin, and A. J. Epstein, Phys. Rev. B 74, 235219 (2006).

${ }^{22}$ J. M. Ziman, Principles of the Theory of Solids (Cambridge University Press, Cambridge, 1964).

${ }^{23}$ N. W. Ashcroft and N. D. Mermin, Solid State Physics (Saunders, New York, 1976).

${ }^{24}$ W. P. Lee, K. R. Brenneman, A. D. Gudmundsdottir, M. S. Platz, P. K. Kahol, A. P. Monkman, and A. J. Epstein, Synth. Met. 101, 819 (1999); P. K. Kahol, R. P. Perera, K. K. Satheesh Kumar, S. Geetha, and D. C. Trivedi, Solid State Commun. 125, 369 (2003).

${ }^{25}$ J. Joo, J. K. Lee, J. K. Hong, J. S. Baeck, W. P. Lee, A. J. Epstein, K. S. Jang, S. J. Suh, and E. J. Oh, Macromolecules 31, 479 (1998); J. Joo, J. K. Lee, J. S. Baeck, K. H. Kim, E. J. Oh, and A. J. Epstein, Synth. Met. 117, 45 (2001).

${ }^{26}$ S. Newman, Electrochemical Systems (Prentice-Hall, Englewoods Cliffs, NY, 1991).

${ }^{27}$ R. Kötz and M. Karlen, Electrochim. Acta 45, 2483 (2000).

${ }^{28}$ R. P. Buck and C. Mundt, Electrochim. Acta 44, 1999 (1999).

${ }^{29}$ G. Paasch, Synth. Met. 119, 233 (2001).

${ }^{30}$ S. M. Sze, Physics of Semiconductor Devices, 2nd ed. (Wiley, New York, 1981).

${ }^{31}$ A. N. Aleshin, H. Sandberg, and H. Stubb, Synth. Met. 121, 1449 (2001); C. Tanase, E. J. Meijer, P. W. M. Blom, and D. M. de Leeuw, Phys. Rev. Lett. 91, 216601 (2003).

${ }^{32}$ I. N. Hulea, H. B. Brom, A. J. Houtepen, D. Vanmaekelbergh, J. J. Kelly, and E. A. Meulenkamp, Phys. Rev. Lett. 93, 166601 (2004).

${ }^{33}$ H. Shimotani, G. Diguet, and Y. Iwasa, Appl. Phys. Lett. 86, 022104 (2005).

${ }^{34}$ G. Horowitz, Adv. Math. 10, 365 (1998).

${ }^{35}$ P. Stallinga and H. L. Gomes, Synth. Met. 156, 1305 (2006).

${ }^{36}$ M. Lapkowski and A. Pron, Synth. Met. 110, 79 (2000).

${ }^{37}$ Th. Johansson, L. A. A. Petersson, and O. Inganas, Synth. Met. 129, 269 (2002). 